\title{
Metabolite and functional profile of patients with cervical spondylotic myelopathy
}

\author{
Izabela Aleksanderek, PhD, ${ }^{1,2}$ Todd K. Stevens, PhD, ${ }^{2}$ Sandy Goncalves, MSc, ${ }^{1,2}$ \\ Robert Bartha, PhD, 1,2 and Neil Duggal, MD1,3

\begin{abstract}
1Department of Medical Biophysics and ${ }^{2}$ Centre for Functional and Metabolic Mapping, Robarts Research Institute, Western University; and ${ }^{3}$ Department of Clinical Neurological Sciences, University Hospital, London Health Sciences Centre, London, Ontario, Canada
\end{abstract}

OBJECTIVE The goal of this study was to compare the recovery of neuronal metabolism and functional reorganization in the primary motor cortex (M1) between mild and moderate cervical spondylotic myelopathy (CSM) following surgical intervention.

METHODS Twenty-eight patients with CSM underwent 3-T MRI scans that included spectroscopy and functional MRI, before surgery and 6 months postsurgery. The classification of severity was based on the modified Japanese Orthopaedic Association questionnaire. Mild and moderate myelopathy were defined by modified Japanese Orthopaedic Association scores $>12$ of $18(n=15)$ and $9-12(n=13)$, respectively. Ten healthy control subjects underwent 2 MRI scans 6 months apart. Metabolite levels were measured in the M1 contralateral to the greater deficit side in patients with CSM and on both sides in the controls. Motor function was assessed using a right finger-tapping paradigm and analyzed with BrainVoyager QX.

RESULTS Patients with mild CSM had a lower preoperative $N$-acetylaspartate to creatine (NAA/Cr) ratio compared with moderate CSM, suggesting mitochondrial dysfunction. Postsurgery, NAA/Cr in moderate CSM decreased to the levels observed in mild CSM. Preoperatively, patients with mild CSM had a larger volume of activation (VOA) in the M1 than those with moderate CSM. Postoperatively, the VOAs were comparable between the mild and moderate CSM groups and had shifted toward the primary sensory cortex.

CONCLUSIONS The NAA/Cr ratio and VOA size in the M1 can be used to discriminate between mild and moderate CSM. Postsurgery, the metabolite profile of the M1 did not recover in either group, despite significant clinical improvement. The authors proposed that metabolic impairment in the M1 may trigger the recruitment of adjacent healthy cortex to achieve functional recovery.

https://thejns.org/doi/abs/10.3171/2016.9.SPINE151507

KEY WORDS cervical spondylotic myelopathy; functional MRI; magnetic resonance spectroscopy; $N$-acetylaspartate; brain plasticity

$\mathrm{C}$ ERVICAL spondylotic myelopathy (CSM) can present abruptly (with severe symptoms of neurological impairment) or insidiously (with a slow, stepwise deterioration).$^{10}$ The benefits of surgery in patients with CSM who have mild symptoms are controversial, because some studies suggest that these patients can stabilize if treated conservatively, whereas others suggest that surgery improves functional outcomes. ${ }^{11,18,23}$ In light of these conflicting data, there is a need for noninvasive imaging biomarkers that could help optimize the selection of patients with CSM for surgical treatment.
The literature has focused on identifying predictors of postoperative outcomes in patients with CSM by examining local changes in the spinal cord. ${ }^{23}$ Recent work by our group has demonstrated that CSM also produces cortical metabolic and functional changes. ${ }^{9,20}$ Specifically, decreased $N$-acetylaspartate (NAA) to creatine $(\mathrm{Cr})$ ratio measured by MR spectroscopy (MRS) was observed in the primary motor cortex (M1), suggesting neuronal impairment and/or altered energy metabolism. ${ }^{20}$ Functional MRI (fMRI) studies have demonstrated cortical reorganization in response to spinal cord compression and sur-

ABBREVIATIONS BA = Brodmann area; $C O G=$ center of gravity; $\mathrm{Cr}=$ creatine; $\mathrm{CSM}=$ cervical spondylotic myelopathy; $\mathrm{FDR}=$ false discovery rate; fMRI = functional MRI; $\mathrm{mJOA}=$ modified Japanese Orthopaedic Association; MRS = MR spectroscopy; M1 = primary motor cortex; NAA = N-acetylaspartate; VOA = volume of activation. SUBMITTED December 21, 2015. ACCEPTED September 12, 2016.

INCLUDE WHEN CITING Published online February 3, 2017; DOI: 10.3171/2016.9.SPINE151507. 
gery. ${ }^{9}$ This evidence suggests that MRS and fMRI are able to detect cortical neuronal and functional changes distal to the site of injury, thus providing imaging correlates of clinical surgical outcome.

The goal of this longitudinal study was to compare the distinct metabolite and functional profiles in the M1 of patients with mild and moderate CSM before and after decompressive surgery. We hypothesized that compared with mild CSM, patients with moderate CSM would have more pronounced preoperative reductions and greatest recovery following surgery, in both $\mathrm{NAA} / \mathrm{Cr}$ and functional activation of the M1.

\section{Methods}

\section{Experimental/Study Design}

Twenty-eight patients with CSM and 10 healthy control subjects with no history of neurological disorders were recruited. The inclusion and exclusion criteria were established prospectively. Subjects were included if symptoms manifested within 1 year prior to their initial clinic visit, they could tolerate an MRI scan, and they were $>18$ years of age. Exclusion criteria included any metal implants (aneurysm clips and so on), claustrophobia, pregnancy, nerve root symptoms or damage, any other neurological disorders such as cerebral palsy, or a history of trauma. The subjects were not randomly assigned and the investigators were not blinded in this study.

The objective of this study was to compare the distinct metabolite and functional profiles in the M1 of patients with mild and moderate CSM before and after decompressive surgery. We hypothesized that compared with mild CSM, patients with moderate CSM would have more pronounced preoperative reductions and greatest recovery following surgery, in both NAA/Cr and functional activation of the M1. The MRI scanning protocol included the acquisition of anatomical images, fMRI, and ${ }^{1} \mathrm{H}$ spectroscopy, and was performed once during each session. Each patient and control had 2 sessions, 6 months apart. Primary and secondary end points were selected prospectively. Primary end points included the changes in metabolite concentrations and functional activations. The secondary end points were the functional improvements shown by clinical assessments.

\section{Clinical Information}

Twenty-eight patients with CSM were recruited. Each patient participated in 3-T MRI sessions, one preoperatively and another 6 months after surgical decompression. Ten healthy control subjects with no history of neurological disorders were also recruited and underwent 2 MRI scans 6 months apart. Informed written consent was obtained from all participants, and the study was approved by the University of Western Ontario Health Sciences Research Ethics Board. Each patient and control subject completed the disease-specific modified Japanese Orthopaedic Association (mJOA) scale at each visit. ${ }^{4}$ Mild CSM $(\mathrm{n}=15)$ was defined as having an mJOA score $>12$ of 18 , whereas patients with moderate CSM $(n=13)$ presented with an mJOA score between 9 and 12 at the initial visit. ${ }^{24}$ There were no severe CSM cases recruited in this study (mJOA score $<9){ }^{24}$

\section{Anatomical Data Acquisition}

All imaging data were acquired using a 3-T Siemens MAGNETOM Tim Trio MRI, using a 12-channel head coil with a neck and spine array. Each examination included the acquisition of sagittal T1-weighted inversionprepared (TI $900 \mathrm{msec}$ ) 3D-magnetization-prepared rapid acquisition gradient echo (MPRAGE) anatomical images (192 slices, 1-mm isotropic resolution, TR/TE 2300/3.42 msec) that covered the entire brain and produced high gray matter/white matter contrast.

\section{Data Acquisition by MRS and Analysis}

Before imaging, an automated global shimming procedure using first- and second-order shims was performed to optimize the magnetic field over the imaging volume of interest. The precentral knob, a reliable landmark that identifies the motor hand function in the precentral gyrus under normal and pathological conditions, was used to guide the placement of a 20 -mm isotropic MRS voxel. ${ }^{27}$ The voxel was placed in the motor cortex contralateral to the greater deficit side in the group of patients with CSM ( $\mathrm{n}=12$ on right side and $\mathrm{n}=16$ on left side) as previously described,$^{13}$ whereas controls had a separate voxel placed on each side of the motor cortex.

Spectroscopic data were acquired using a point-resolved spectroscopy (PRESS) acquisition sequence (TR/ TE 2000/135 msec, voxel size $8 \mathrm{~cm}^{3}$, 192 averages with water suppression, 8 averages without suppression) and postprocessed with combined QUALITY deconvolution $^{7}$ to restore the Lorentzian line shape and eddy current correction. ${ }^{1}$ Following line shape correction, any remaining unsuppressed water between 4.1 and $5.1 \mathrm{ppm}$ was removed from the metabolite spectra using a Hankel singular-value decomposition that required no prior knowledge. ${ }^{19}$

A Levenberg-Marquardt minimization routine incorporating a template of prior knowledge of metabolite line shapes was used to fit the resultant metabolite spectra in the time domain. ${ }^{2,19}$ Following brain segmentation using the FMRIB Software Library (FSL) library ${ }_{17}^{17}$ all metabolite levels were corrected for $T_{1}$ and $T_{2}$ relaxation specific to each metabolite signal as well as to the voxel-specific composition of gray matter, white matter, and CSF. NAA and $\mathrm{Cr}$ were examined. The NAA/Cr ratio was calculated and compared between groups. The NAA/Cr ratio eliminates potential differences in tissue partial volume between voxels because neither NAA nor $\mathrm{Cr}$ is present in appreciable concentrations in CSF.

\section{Data Acquisition by fMRI and Analysis}

Each functional brain volume was acquired using an interleaved echo-planar imaging pulse sequence (integrated Parallel Imaging Technique [iPAT] $=2,240 \times 240$ acquisition matrix, 45 slices/volume, 3-mm isotropic resolution, TR/TE 2500/30 msec, flip angle $90^{\circ}$ ).

To test the motor pathway during fMRI, participants were instructed to press a button placed on their thumb with all 4 fingers simultaneously, followed by an extension upward to a box surrounding the button in their right hand. The box ensured that each participant lifted their 
fingers at the same angle by touching the top of the box prior to the next tap. The movement rate of the repetitive task is known to affect cerebral activation; ${ }^{5}$ therefore, a standard block design was used in which participants received visual cues during alternating 30 -second intervals of rest and activity. The participants tapped every 3 seconds for the 30 -second interval. Participants received training prior to the fMRI session to reinforce the standardization and to reduce learning effects during the imaging session.

Image analysis was completed using BrainVoyager QX (Brain Innovation B.V.). Anatomical images were transformed to Talairach space for group analysis. Preprocessing of functional images included 3D motion correction (trilinear/sinc interpolation where only images with small motion-related effects [ $2 \mathrm{~mm}$ movement] were included), spatial smoothing (6 $\mathrm{mm}$ full width at half maximum gaussian filter), slice scan-time correction, and linear trend removal. Functional data were then registered to the anatomical images in Talairach space, and a 4D volume time course file was created for each subject.

A general linear model and random-effects analysis were used to create individual and group statistical parametric maps of brain activation. A boxcar function coincident with the paradigm with a double-Gamma hemodynamic response function was used as a predictor of the fMRI response to the motor task. ANOVA tests were used to identify significant clusters of activation in each group at each time point, which were set to a threshold using a corrected value of $p<0.001$ controlling the false discovery rate (FDR). Resulting contrast maps were set to a threshold of $\mathrm{p}<0.05$ and corrected for multiple comparisons using the cluster size correction plug-in of BrainVoyager. ${ }^{12}$ For all contrasts, a volume of activation (VOA), corrected $\mathrm{p}$ value, and Talairach coordinates of the center of gravity (COG) of the activation were examined. Talairach Daemon Client 2.0 (University of Texas Health Science Center) was used to convert the Talairach coordinates to their corresponding Brodmann areas (BAs). ${ }^{25}$

\section{Statistical Analysis}

All data are presented as the group mean followed by the standard deviation. The mJOA scores were compared between mild and moderate CSM groups using a 2-tailed Student's t-test at both time points. Student's ttest was also used to identify the significant differences in metabolite ratios between the control, patients with mild CSM, and patients with moderate CSM groups at pre- and postoperative time points. Next, a paired t-test was used to detect the effects of surgery on the CSM groups. Subjects with missing data were excluded from the paired analysis. All statistical tests were 2 sided, with significance set at an alpha level of 0.05 . Pearson correlations were used to examine associations between levels of NAA/Cr and mJOA scores ( $p<0.05$ considered significant).

\section{Results \\ Clinical Data}

We recruited 15 patients with mild CSM with a mean age of 50.1 years ( $\mathrm{SD} \pm 12$ years; 13 men) and 13 patients
TABLE 1. Demographic and clinical data of patients with CSM and healthy control subjects

\begin{tabular}{|c|c|c|c|}
\hline \multirow[b]{2}{*}{ Variable } & \multicolumn{3}{|c|}{ Moderate } \\
\hline & Mild CSM & CSM & Control \\
\hline No. & 15 & 13 & 10 \\
\hline Age in yrs, mean $\pm S D$ & $50.1 \pm 12$ & $53.0 \pm 9$ & $48.0 \pm 12$ \\
\hline Sex, M/F & 13:2 & $8: 5$ & $5: 5$ \\
\hline Hand dominance, rt/lt & $13: 2$ & 13:0 & $10: 0$ \\
\hline $\begin{array}{l}\text { Duration of symptoms in } \\
\text { mos, mean } \pm \text { SD }\end{array}$ & $7.9 \pm 8.2$ & $8.6 \pm 5.5$ & - \\
\hline $\begin{array}{l}\text { Signal change on T2- } \\
\text { weighted MR image at } \\
\text { site of compression }\end{array}$ & $14 / 15 \mathrm{pts}$ & $10 / 13$ pts & - \\
\hline \multicolumn{4}{|l|}{ Surgery } \\
\hline 1 level, no. (\%) & $12(80)$ & $7(54)$ & - \\
\hline 2 level, no. (\%) & $3(20)$ & $6(46)$ & - \\
\hline \multicolumn{4}{|l|}{$\begin{array}{l}\text { Length of follow-up in days, } \\
\text { mean } \pm \text { SD }\end{array}$} \\
\hline $\begin{array}{l}\text { Surgery (CSM) or } \\
\text { baseline (controls) } \\
\& 6 \text {-mo scan }\end{array}$ & $188 \pm 61$ & $201 \pm 64$ & $176 \pm 48$ \\
\hline \multicolumn{4}{|l|}{ mJOA score, mean \pm SD } \\
\hline Preop & $15.1 \pm 1.2$ & $10.6 \pm 1.5$ & $18 \pm 0$ \\
\hline Postop & $16.3 \pm 1.2$ & $15.4 \pm 1.9$ & $18 \pm 0$ \\
\hline
\end{tabular}

Pts $=$ patients $;-=$ not applicable.

with moderate CSM with a mean age of 53.0 years (SD \pm 9 years; 8 men). Ten healthy control subjects of similar age were recruited $(48.0 \pm 12$ years, 5 men). The average lengths of follow-up were $188 \pm 61$ days, $201 \pm 64$ days, and $176 \pm 48$ days for the mild CSM, moderate CSM, and control groups, respectively. The 3 groups did not differ with respect to age, sex, or length of follow-up. The mild and moderate CSM groups did not differ in their duration of self-reported symptoms or the presence of signal change at the site of compression on T2-weighted images. However, there were more 2-level decompression surgeries performed in the moderate CSM group. In the mild CSM group, NAA/Cr data were not available in 2 subjects at baseline and in 1 subject at follow-up due to incorrect voxel size or position. In the moderate CSM group, NAA/ $\mathrm{Cr}$ data were not available in 2 subjects at baseline, also due to incorrect voxel position. Demographic data and clinical characteristics of patients with CSM and healthy control subjects are summarized in Table 1.

In the 6 months after surgery, the mJOA scores increased for all patients with CSM except 1 , who had a decrease of 1 point (Fig. 1). Patients with mild CSM with an average preoperative mJOA score of $15.1 \pm 1.2$ improved to $16.3 \pm 1.2$ postoperatively $(p=0.002)$. The mJOA score in the moderate CSM group increased from $10.6 \pm 1.5$ preoperatively to $15.4 \pm 1.9$ postoperatively $(\mathrm{p}<0.0001)$. The mild and moderate CSM groups were significantly different preoperatively $(\mathrm{p}<0.0001)$ but recovered to the same postoperative score $(\mathrm{p}=0.14)$. For all control subjects, a perfect mJOA score of 18 was recorded at both visits (Table 1). 


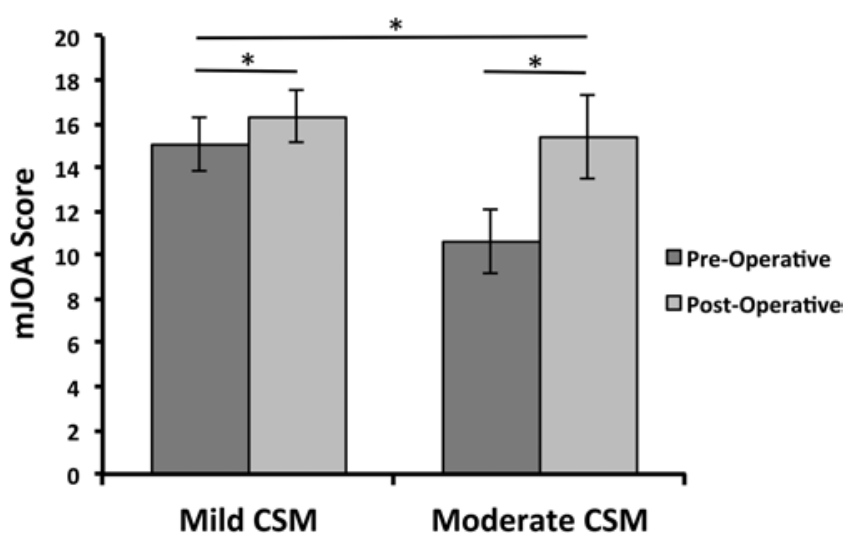

FIG. 1. Bar graph showing the mean pre- and postoperative mJOA questionnaire scores for patients in the mild and moderate CSM groups. A perfect mJOA score of 18 represents no dysfunction. Error bars represent the standard deviation. ${ }^{*} p<0.05$.

\section{Metabolite Profile of Control Subjects}

The control group presented with no changes in the $\mathrm{NAA} / \mathrm{Cr}$ ratio over time (baseline $1.91 \pm 0.15$, follow-up $1.90 \pm 0.29 ; \mathrm{p}=0.89, \mathrm{n}=10$ ).

\section{Metabolite Profile of Patients With Mild CSM}

Among patients in the mild CSM group, the preoperative NAA/Cr ratio of $1.55 \pm 0.46$ did not change following surgery $(1.44 \pm 0.33 ; \mathrm{p}=0.50, \mathrm{n}=12)$, but was significantly decreased compared with the control subjects $(\mathrm{n}=$ 10) at preoperative $(\mathrm{p}=0.02, \mathrm{n}=13)$ and postoperative $(\mathrm{p}$ $=0.0004, \mathrm{n}=14)$ time points $($ Fig. 2$)$.

\section{Metabolite Profile of Patients With Moderate CSM}

Patients with moderate CSM presented with a preoperative NAA/Cr ratio of $1.94 \pm 0.27$, which significantly decreased to $1.51 \pm 0.39(\mathrm{p}=0.03, \mathrm{n}=11)$ postsurgery (Fig. 2). The postoperative NAA/Cr was significantly lower in patients with moderate CSM compared with control subjects $(\mathrm{p}=0.01, \mathrm{n}=13)$.

\section{Patients With Mild CSM Versus Moderate CSM}

There is an important distinction between patients with mild and moderate CSM. Patients with mild CSM (n = 13) presented with a much lower preoperative NAA/Cr ratio ( $\mathrm{p}=0.02$; Fig. 2$)$ compared with those with moderate CSM $(\mathrm{n}=11)$. Following surgery, the NAA/Cr ratio in patients with moderate CSM decreased such that patients with mild and moderate CSM had similar NAA/Cr ratios. There was no significant association between the length of illness and either baseline NAA/Cr levels or the change in NAA/Cr levels after surgery. When examining all subjects along a continuum, there was a trend toward an association between baseline NAA/Cr and baseline mJOA score $(\mathrm{r}=-0.32, \mathrm{p}=0.06)$, baseline NAA/Cr and the change in mJOA score $(r=0.29, p=0.08)$, and the change in NAA/ $\mathrm{Cr}$ over 6 months with the change in mJOA score $(\mathrm{r}=$ $-0.27, \mathrm{p}=0.10$ ).

\section{Motor Function Assessed by fMRI}

The control subjects showed no differences in activa-

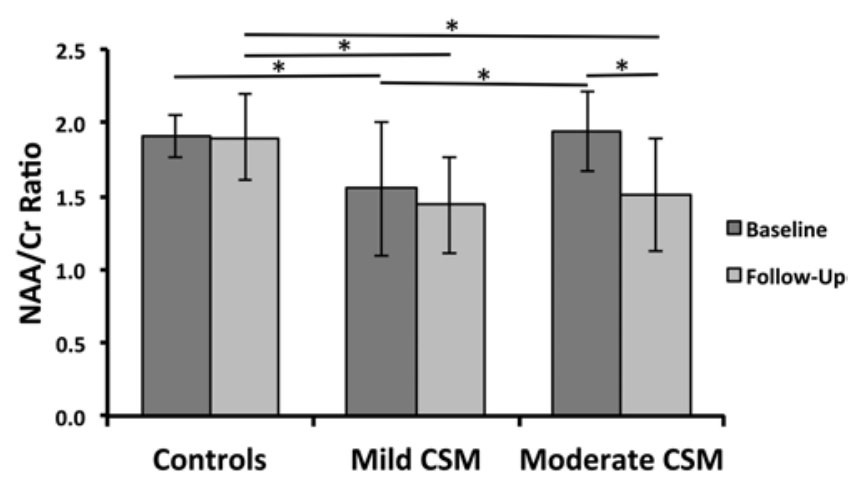

FIG. 2. Mean NAA/Cr concentrations. Mean NAA/Cr metabolite concentrations at baseline and at 6-month follow-up in the control subjects, and at pre- and postoperative time points in the mild and moderate CSM groups. Error bars represent the standard deviation. ${ }^{*} p<0.05$.

tion associated with right-hand tapping between the baseline and the 6-month repeat scans. In addition, there were no activation differences between controls and the mild or moderate CSM group at either time point. Preoperatively, patients with mild CSM had a larger VOA (Fig. 3) compared with those with moderate CSM ( $\mathrm{p}=0.05$; minimum cluster size 118; COG [27, -42, 58], left parietal lobe, postcentral gyrus, BA 5). Following surgery, the VOAs centered on BA 3 in patients with mild and moderate CSM did not differ significantly (mild CSM: FDR < 0.001; $\mathrm{p}<$ 0.000014; COG [38, -30, 53], left parietal lobe, postcentral gyrus, BA 3, and moderate CSM: FDR < 0.001; p < 0.000006; COG [37, -28, 54], left parietal lobe, postcentral gyrus, BA 3). There was an increase in the VOA in moderate CSM following surgery using an uncorrected $p$ value; however the change did not reach the threshold for significance after FDR correction.

\section{Discussion}

The overall goal of this study was to compare and contrast the effects of mild and moderate CSM on the neuronal metabolism and functional activity of the M1. The need for surgical intervention for patients with clinical evidence of mild CSM has become increasingly controversial. Our findings demonstrated a lower NAA/Cr ratio in the hand area of the M1 in patients with mild CSM compared with healthy control subjects and patients with moderate CSM. Following successful surgery (and despite clinical improvement), NAA/Cr levels did not recover in mild CSM. The patients with moderate CSM, who had significantly worse preoperative mJOA scores and demonstrated the largest interval improvement following surgery, demonstrated a decline in NAA/Cr levels following surgery.

In conjunction with evaluation of tissue metabolism, fMRI was used to evaluate M1 function pre- and postoperatively. Prior to surgery, in the setting of low NAA/Cr levels in the mild CSM group, but normal NAA/Cr levels in the moderate CSM group, we found a larger VOA within the sensorimotor cortex of mild CSM compared with moderate CSM. Consistent with a previous study showing greater M1 activation in patients with CSM compared with controls, ${ }^{9}$ our fMRI findings suggest that in the mild CSM 


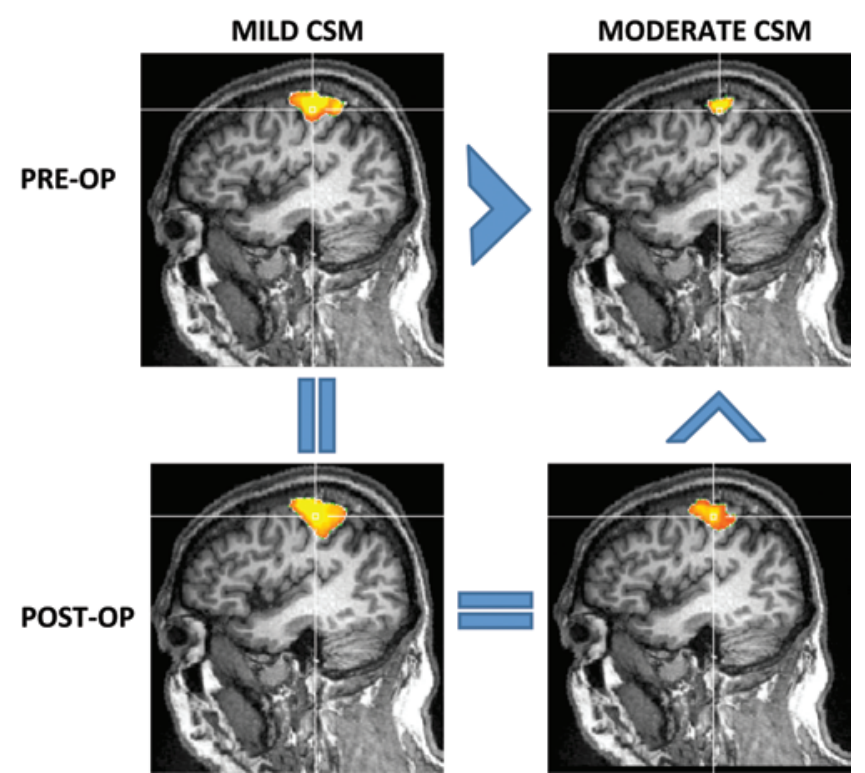

FIG. 3. Volumes of activation in patients with mild and moderate CSM. The VOAs for patients in the mild CSM (left) and the moderate CSM (right) groups are shown. The preoperative activation (upper) shows that the mild CSM group had significantly larger activation near the M1 compared with the moderate CSM group. Following surgery (lower), both groups had equal VOAs shifted toward BA 3 , the primary somatosensory cortex. The patients with moderate CSM showed an increase in the VOA after surgery using an uncorrected $p$ value; however, this increase did not remain significant following FDR correction.

group, there was successful recruitment of surrounding cortex to enhance motor task performance prior to surgery.

Following surgery in the moderate CSM group, functional improvement was accompanied by decreased NAA/ $\mathrm{Cr}$ while fMRI-measured VOA equalized to that observed in the mild CSM group. These distinct patterns of remote injury of the sensorimotor cortex in patients with mild and moderate CSM could underlie the natural history of the disease. These results also suggest that therapies designed to enhance brain plasticity could provide functional benefits to patients with CSM both prior to and following surgery. ${ }^{8,15}$

To our knowledge, this is the first study to describe a clear distinction between mild and moderate CSM with respect to preoperative NAA/Cr concentrations in the motor cortex. NAA has one of the highest concentrations of all free amino acids in the brain and is found primarily in the neurons of the CNS. ${ }^{22}$ Reduced levels of NAA/Cr imply neuronal loss or metabolic impairment in the neuronal mitochondria. ${ }^{22}$ Decreased NAA/Cr in the motor cortex 6 months after surgery could be due to injury to the cord, consistent with previous studies in animal models that have shown atrophy ${ }^{6}$ or shrinkage of pyramidal neurons in the motor cortex following axotomy. 3,26

We previously reported decreased NAA/Cr levels in the motor cortex of patients with CSM compared with healthy control subjects. ${ }^{20}$ Holly et al. also found a decreased NAA/Cr ratio at the C-2 level of the spinal cord in patients with CSM, suggesting axonal or neuronal loss. ${ }^{16}$ In the current study, the measured NAA/Cr levels were lower in mild CSM compared with moderate CSM, sug- gesting that metabolic impairment in the neuronal mitochondria may be associated with mild functional impairment. Although counterintuitive, previous work has shown that decreased mitochondrial function can lead to synaptic dysfunction that may in turn trigger cortical reorganization and recruitment of healthy regions in the primary sensory cortex..$^{21}$

We speculate that this process could explain why neurological function was better preserved in the mild CSM group at baseline; these patients had already recruited adjacent cortex, as indicated by a larger VOA at baseline compared with the patients with moderate CSM. This result is also consistent with a previous study that found that patients with spinal cord compression had an increased VOA within the motor cortex compared with controls. ${ }^{9}$ In contrast, the moderate CSM group, which had normal NAA/Cr levels but profound neurological deficits preoperatively, demonstrated small preoperative VOAs. This evidence suggests that prior to surgery, the moderate CSM group had a limited compensatory ability for reorganization, accounting for the poor neurological scores.

Further analysis demonstrated trends observed across all subjects in the levels of NAA/Cr and mJOA scores, confirming that people with lower baseline NAA/Cr levels had greater function at baseline, people with high NAA/Cr levels at baseline had the greatest improvement in mJOA scores 6 months after surgery, and people whose NAA/Cr levels decreased the most after surgery had the greatest improvement in function. These trends are consistent with our observations and interpretation of the changes in the mild and moderate CSM groups.

Following surgery, both the patients with mild and moderate CSM demonstrated interval functional improvement. However, a larger mJOA improvement was observed in the moderate CSM group; namely, patients with the most severe symptoms demonstrated the greatest measurable clinical improvement. One possible explanation for this finding is that patients with mild CSM can describe mild sensory loss on the mJOA scale, such as paresthesia, which may persist despite successful surgical treatment. An additional explanation for the limited improvement in the mJOA score in patients with mild CSM may be the ceiling effect for this patient-derived outcome measure, where patients with mild disease experience a smaller absolute change in score as they approach a perfect mJOA score. Six months after surgery, both groups had mJOA scores reflecting mild neurological disability. Surgical intervention did not reverse the low NAA/Cr levels in the mild CSM group, nor did it preserve the normal levels of NAA/ $\mathrm{Cr}$ in the moderate CSM group; instead, NAA/Cr levels declined following surgery in the moderate CSM group.

In light of the low NAA/Cr ratio in mild CSM and the decrease in the NAA/Cr ratio observed in moderate CSM 6 months after surgery, adaptive plasticity in response to mitochondrial and synaptic dysfunction is a possible explanation for the observed neurological recovery. The observed increase in the VOA in moderate CSM (prior to FDR correction) is consistent with this notion. Prior studies have also shown that neurological recovery following surgical decompression is associated with cortical reorganization. ${ }^{8,9,15}$ The exact mechanism by which cortical 
reorganization occurs is not completely understood, but it has been suggested that it may occur by modification of preexisting connections and/or the development of new circuitry intended to preserve neurological functioning. ${ }^{15}$ Patients who have suffered stroke have also presented with spontaneous recovery that occurs due to cortical plasticity, with the best recovery resulting from reorganization in the damaged hemisphere. ${ }^{21}$

In the current study, one possible explanation for the posterior shift in activation is that the corticospinal axons originating in the primary sensory cortex are preserved because they are less vulnerable to injury than the axons originating in the M1. This advantage exists because the fibers in the primary sensory cortex run more medial and posterior in the spinal cord ${ }^{8,14}$ Another potential explanation for the observed increase in activation volume in the sensory cortex of the moderate CSM group 6 months after surgery is an increase in sensory function. Future studies should include metabolite level measurements in both the primary motor and sensory cortices to evaluate potential changes in NAA due to the reorganization.

Several limitations to this study must be taken into consideration. The grouping of subjects into the mild and moderate CSM groups was accomplished using the mJOA score. Although this grouping has been previously used, ${ }^{24}$ we acknowledge that the classification of mild and moderate CSM is somewhat artificial and other groupings are also possible. However, such a classification was applied in the current study to aid in understanding whether patients with early or subtle signs of cervical myelopathy may benefit from surgery. Given the small sample size in this study, the smallest detectable difference in NAA/Cr with $80 \%$ power and $\alpha=0.05$ was 0.35 in the mild CSM group $(n=$ 12 with pre- and postoperative data, average standard deviation of NAA/ $\mathrm{Cr}=0.40$ ) and 0.31 in the moderate CSM group $(\mathrm{n}=11$ with pre- and postoperative data, average standard deviation of NAA/Cr $=0.33$ ). It should be noted that more participants in the moderate CSM group (46\%) underwent 2-level decompression compared with the mild CSM group (20\%).

\section{Conclusions}

As a unique condition of reversible spinal cord injury, CSM can be exploited to understand the link between brain metabolic dysfunction and synaptic activity and remodeling. The current study found distinct metabolite and functional profiles in the motor cortex that discriminate between patients with mild and moderate CSM prior to surgery. Following surgery, the NAA/Cr ratio in the motor cortex decreased significantly in moderate CSM, but was accompanied by neurological improvement and increased brain activation equal to that observed in patients with mild CSM. We propose that mitochondrial dysfunction, evidenced by low levels of NAA/Cr, is the primary trigger for cortical reorganization and recruitment leading to functional improvement.

\section{Acknowledgments}

We would like to thank all participants for their contributions to this project. This work was funded by the Physicians Services
Incorporated Foundation (07-37 to N.D. and R.B.), the Canadian Institutes of Health Research (231560 to N.D. and R.B.), and the Academic Development Fund Small Grant (SG11-10 to N.D., R.B., and I.A.).

\section{References}

1. Bartha R, Drost DJ, Menon RS, Williamson PC: Spectroscopic lineshape correction by QUECC: combined QUALITY deconvolution and eddy current correction. Magn Reson Med 44:641-645, 2000

2. Bartha R, Drost DJ, Williamson PC: Factors affecting the quantification of short echo in-vivo $1 \mathrm{H}$ MR spectra: prior knowledge, peak elimination, and filtering. NMR Biomed 12:205-216, 1999

3. Beaud ML, Schmidlin E, Wannier T, Freund P, Bloch J, Mir A, et al: Anti-Nogo-A antibody treatment does not prevent cell body shrinkage in the motor cortex in adult monkeys subjected to unilateral cervical cord lesion. BMC Neurosci 9:5, 2008

4. Benzel EC, Lancon J, Kesterson L, Hadden T: Cervical laminectomy and dentate ligament section for cervical spondylotic myelopathy. J Spinal Disord 4:286-295, 1991

5. Blinkenberg M, Bonde C, Holm S, Svarer C, Andersen J, Paulson OB, et al: Rate dependence of regional cerebral activation during performance of a repetitive motor task: a PET study. J Cereb Blood Flow Metab 16:794-803, 1996

6. Brock JH, Rosenzweig ES, Blesch A, Moseanko R, Havton LA, Edgerton VR, et al: Local and remote growth factor effects after primate spinal cord injury. J Neurosci 30:97289737, 2010

7. de Graaf AA, van Dijk JE, Bovée WM: QUALITY: quantification improvement by converting lineshapes to the Lorentzian type. Magn Reson Med 13:343-357, 1990

8. Dong Y, Holly LT, Albistegui-Dubois R, Yan X, Marehbian J, Newton JM, et al: Compensatory cerebral adaptations before and evolving changes after surgical decompression in cervical spondylotic myelopathy. J Neurosurg Spine 9:538-551, 2008

9. Duggal N, Rabin D, Bartha R, Barry RL, Gati JS, Kowalczyk I, et al: Brain reorganization in patients with spinal cord compression evaluated using fMRI. Neurology 74:1048-1054, 2010

10. Fehlings MG, Skaf G: A review of the pathophysiology of cervical spondylotic myelopathy with insights for potential novel mechanisms drawn from traumatic spinal cord injury. Spine (Phila Pa 1976) 23:2730-2737, 1998

11. Fehlings MG, Wilson JR, Kopjar B, Yoon ST, Arnold PM, Massicotte EM, et al: Efficacy and safety of surgical decompression in patients with cervical spondylotic myelopathy: results of the AOSpine North America prospective multicenter study. J Bone Joint Surg Am 95:1651-1658, 2013

12. Forman SD, Cohen JD, Fitzgerald M, Eddy WF, Mintun MA, Noll DC: Improved assessment of significant activation in functional magnetic resonance imaging (fMRI): use of a cluster-size threshold. Magn Reson Med 33:636-647, 1995

13. Goncalves S, Stevens TK, Doyle-Pettypiece P, Bartha R, Duggal N: N-acetylaspartate in the motor and sensory cortices following functional recovery after surgery for cervical spondylotic myelopathy. J Neurosurg Spine 25:436-443, 2016

14. Green JB, Sora E, Bialy Y, Ricamato A, Thatcher RW: Cortical motor reorganization after paraplegia: an EEG study. Neurology 53:736-743, 1999

15. Holly LT, Dong Y, Albistegui-DuBois R, Marehbian J, Dobkin B: Cortical reorganization in patients with cervical spondylotic myelopathy. J Neurosurg Spine 6:544-551, 2007

16. Holly LT, Freitas B, McArthur DL, Salamon N: Proton magnetic resonance spectroscopy to evaluate spinal cord axonal 
injury in cervical spondylotic myelopathy. J Neurosurg Spine 10:194-200, 2009

17. Jenkinson M, Beckmann CF, Behrens TE, Woolrich MW, Smith SM: FSL. Neuroimage 62:782-790, 2012

18. Kadaňka Z, Bednařík J, Novotný O, Urbánek I, Dušek L: Cervical spondylotic myelopathy: conservative versus surgical treatment after 10 years. Eur Spine J 20:1533-1538, 2011

19. Kassem MN, Bartha R: Quantitative proton short-echo-time LASER spectroscopy of normal human white matter and hippocampus at 4 Tesla incorporating macromolecule subtraction. Magn Reson Med 49:918-927, 2003

20. Kowalczyk I, Duggal N, Bartha R: Proton magnetic resonance spectroscopy of the motor cortex in cervical myelopathy. Brain 135:461-468, 2012

21. Mattson MP: Mitochondrial regulation of neuronal plasticity. Neurochem Res 32:707-715, 2007

22. Moffett JR, Ross B, Arun P, Madhavarao CN, Namboodiri AM: N-Acetylaspartate in the CNS: from neurodiagnostics to neurobiology. Prog Neurobiol 81:89-131, 2007

23. Oshima Y, Seichi A, Takeshita K, Chikuda H, Ono T, Baba S, et al: Natural course and prognostic factors in patients with mild cervical spondylotic myelopathy with increased signal intensity on T2-weighted magnetic resonance imaging. Spine (Phila Pa 1976) 37:1909-1913, 2012

24. Revanappa KK, Rajshekhar V: Comparison of Nurick grading system and modified Japanese Orthopaedic Association scoring system in evaluation of patients with cervical spondylotic myelopathy. Eur Spine J 20:1545-1551, 2011

25. Talairach J, Tournoux P: Co-Planar Stereotaxic Atlas of the Human Brain: 3-Dimensional Proportional System: An Approach to Cerebral Imaging. Stuttgart: Thieme, 1988

26. Wannier T, Schmidlin E, Bloch J, Rouiller EM: A unilateral section of the corticospinal tract at cervical level in primate does not lead to measurable cell loss in motor cortex. J Neurotrauma 22:703-717, 2005

27. Yousry TA, Schmid UD, Alkadhi H, Schmidt D, Peraud
A, Buettner A, et al: Localization of the motor hand area to a knob on the precentral gyrus. A new landmark. Brain 120:141-157, 1997

\section{Disclosures}

The authors report no conflict of interest concerning the materials or methods used in this study or the findings specified in this paper.

\section{Author Contributions}

Conception and design: Duggal, Bartha. Acquisition of data: Aleksanderek, Goncalves. Analysis and interpretation of data: Duggal, Aleksanderek, Stevens, Bartha. Drafting the article: Aleksanderek. Critically revising the article: all authors. Reviewed submitted version of manuscript: all authors. Approved the final version of the manuscript on behalf of all authors: Duggal. Statistical analysis: Aleksanderek. Administrative/technical/material support: Stevens, Goncalves. Study supervision: Duggal, Bartha.

\section{Supplemental Information \\ Previous Presentations}

Portions of this work were presented in poster form at the 23rd Annual Meeting of the International Society for Magnetic Resonance in Medicine, Toronto, Ontario, Canada, May 30 to June 5, 2015.

\section{Correspondence}

Neil Duggal, Department of Clinical Neurological Sciences, Division of Neurosurgery, University Hospital, LHSC, 339 Windermere Rd., London, ON N6A 5A5, Canada. email: neil.duggal@ lhsc.on.ca. 